\title{
EVALUATION OF THE EFFECT OF GARLIC ON THE EXPRESSION OF NF- $\kappa$ B IN DMBA-INDUCED ORAL CARCINOGENESIS IN ALBINO RATS: AN ANIMAL STUDY
}

\author{
Shaimaa Omar Zayed * and Shereen Ali **
}

\begin{abstract}
Background: Nuclear factor-kappa B $(\mathrm{NF}-\kappa \mathrm{B})$ is a key player in the process of carcinogenesis via regulating various steps, thus it is an attractive target for cancer therapy. Many medicinal plants and nutraceuticals can modulate NF- $\mathrm{BB}$ signaling pathway. The aim of this study is to investigate the chemopreventive and immunomodulatory effect of garlic in 7, 12 Dimethylbenz [a] anthracene (DMBA) induced oral carcinogenesis by assessing the expression of NF- $\mathrm{KB}$.

Material and methods: DMBA was used to induce oral cancer in 75 male albino rats. The animals were randomized into five groups ( 1 control and 4 experimental groups). The control group received no DMBA nor garlic extract, the other groups received either DMBA, garlic extract alone or both of them.

Results: NF-кB immunoexpression revealed the highest values in group (2) and group (5) showing a highly significant difference.

Conclusion: The aqueous garlic extract could prevent oral cancer through inhibition of $\mathrm{NF}-\kappa \mathrm{B}$ activation, where its effect is more pronounced in the initiation phase than the promotion and progression phases.
\end{abstract}

KEY WORDS: oral carcinogenesis, garlic, Nuclear Factor-kappa B.

\section{INTRODUCTION}

Oral carcinogenesis involves a chain of multiple stages and events that disrupt the normal regulatory pathways controlling cellular functions such as cell division, differentiation, and cell death. These genetic and epigenetic events create cell phenotype characterized by increased proliferation, loss of cohesion, and ability to invade local tissue as well as metastasis to distant sites (Williams, 2000 and Lin et al., 2012).

* Lecturer, Department of Oral Pathology, Faculty of dentistry, Cairo University.

** Lecturer, Department of Oral Medicine and Periodontology, Faculty of dentistry, Cairo University. Lecturer, Department of Oral Medicine, Periodontology and Oral Radiology, Faculty of dentistry, Misr International University (MIU). 
A major mediator of carcinogenesis is chronic inflammation. Nuclear factor-kappa B (NF- $\mathrm{B}$ ) is the hallmark of the inflammatory response, thus it is a key player in the process of carcinogenesis via regulating various steps starting from initiation ending with metastasis. NF- $\kappa \mathrm{B}$ pathway is activated in all types of cancers including oral cancer where it controls the expression of genes regulating the transformation, survival, proliferation, apoptosis, invasion, angiogenesis, and metastasis of tumor cells (Sarkar et al., 2008; Gupta et al., 2010; Xiao \& $\mathrm{Fu}, 2011$ and Baliga et al., 2015). Thus, the NF-кB signaling pathway is an attractive target for cancer therapy (Aggarwal et al., 2004; Gupta et al., 2010 and Lin et al., 2012).

Currently, there is growing attention for the value of nutraceuticals and medicinal plants in therapeutics due to fewer adverse effects (Arreola et al., 2015). Garlic (Allium sativum Linn.) is one of the most distinguished medicinal plants. The beneficial effect of garlic against multiple systemic diseases, infectious and inflammatory diseases in addition to cancer is well known (Balasanthil et al., 2003; El-Bayoumy et al., 2006; Schafer and Kaschula, 2014 and Arreola et al., 2015).

Garlic can modulate cell signaling and inflammatory pathways; consequently affect tumor development and progression (Balasanthil et al., 2003 and Gupta et al., 2010).

Although many studies focused on the effect of the therapy on cancer cells, the tumor microenvironment is more important in cancer prevention and treatment. Evaluating the effect of the therapy on the tumor microenvironment is done by assessing the expression of targeted molecules (Casey et al., 2015).

The aim of the present study is to evaluate the chemopreventive and immunomodulatory effect of garlic in 7, 12 Dimethylbenz[a]anthracene (DMBA) induced oral carcinogenesis by assessing the expression of NF- $\kappa \mathrm{B}$.

\section{MATERIALS AND METHODS}

\section{Experimental Animals:}

A total of 75 male white albino rats weighing 180-200 grams each were used in the present study. All animals were obtained from the Animal House, Faculty of Medicine, Cairo University and maintained on a basic diet of regular rat chow, distilled water and libitum which is formulated to meet the nutrient needs of rodents.

\section{Chemicals:}

Chemical carcinogen was used to induce oral cancer in rats, for that purpose DMBA was purchased from Sigma Chemical Company (St. Louis, MO, USA).

\section{Induction of oral cancer:}

The induction was made by topical application of $0.5 \%$ DMBA solution in acetone three times per week, using paint brush on the palatal surface of the white albino rats for 22 weeks.

\section{Preparation of garlic extract:}

Aqueous garlic extract was prepared by homogenizing freshly peeled garlic cloves in a small amount of distilled water. The garlic homogenate was placed in ultrasonic shaker for one hour to make extraction of garlic constituents under room temperature (cold extraction). The homogenate was filtered to obtain a clear homogenous filtrate. To adjust a concentration of $25 \mathrm{mg}$ garlic/ml distilled water, the aqueous garlic filtrate was diluted by distilled water to produce the proper concentration (Balasenthil and Nagini, 2000).

\section{Treatment schedule:}

The animals were randomized using random.org into five groups ( 1 control group and 4 experimental groups); 15 animals in each group:

- Group 1 (control): The animals received no treatment. 
- Group 2: The animals received DMBA for 22 weeks.

- Group 3: The animals received intra-gastric administration of aqueous garlic extract three times per week for 22 weeks.

- Group 4: The animals received intra-gastric administration of aqueous garlic extract for two weeks before induction of oral carcinogenesis. After that the animals received DMBA alternating with garlic (each one, three times/week) for 22 weeks.

- Group 5: The animals received DMBA for 22 weeks then received intra-gastric administration of aqueous garlic extract for 8 weeks.

The experiment was terminated at the end of 30 weeks and all animals were sacrificed by cervical dislocation after an overnight fast.

\section{Histopathological and Immunohistochemical Evaluation:}

Fresh tissues were used for histopathological and immunohistochemical examination. Tissues were fixed in $10 \%$ formalin, and then processed for preparing a paraffin block. $5 \mathrm{~mm}$ sections cut on a rotary microtome and stained with conventional Hematoxyline \& Eosin (H\&E) for histopathologic interpretation and immunostained using Cell and Tissue staining kit Horseraddish peroxidase - 3-3' diaminobenzidine (HRP-DAB) system, mouse kit", Mouse-Monoclonal NF-кB Antibody [R\&D systems, Inc. Minneapolis, USA] for evaluation of $\mathrm{NF}-\kappa \mathrm{B} 2$ expression.

\section{Image Analysis:}

An image analyzer computer system using the software Leica Queen 500 was used to examine the histological sections. The immunoreactivity for $\mathrm{NF}-\kappa \mathrm{B}$ was measured in the form of area percentage (area \%) and optical density.

\section{Statistical Analysis}

The data were described as means and standard deviations. The Shapiro-Wilk test was applied to test the normality of the data. ANOVA test was used to assess the differences between the studied groups. The significance level was verified at $\mathrm{P} \leq 0.05$. The results are considered to be statistically significant if p-value was less than 0.05. Computerized statistical package SPSS $16.0 ®$ for Windows (SPSS Inc., Chicago, IL) was used for analyzing the data.

\section{RESULTS}

Histopathological examination of the H\&E stained sections revealed normal keratinized stratified squamous epithelium in both control animals (group 1) and animals that received garlic extract alone (group 3).

In group 2 (rats that received DMBA alone), the histopathological examination revealed squamous cell carcinoma except for two specimens that showed carcinoma insitu with pronounced basilar hyperplasia.

In group 4 (animals that received garlic extract followed by DMBA alternating with garlic), 12 tissue sections revealed keratinized stratified squamous epithelium with some areas of hyperplasia but no signs of dysplasia and 3 tissue sections revealed basilar hyperplasia in some areas but no other signs of dysplasia.

In group 5 (rats that received DMBA followed by garlic), 6 of the H\&E stained sections showed hyperplasia and hyperkeratosis and 9 developed squamous cell carcinoma (Figure 1).

As shown in figure (2), the palatal epithelium of all the studied groups revealed immunopositive expression of NF-кB. However, groups (1) and (3) revealed very mild cytoplasmic NF- $\kappa \mathrm{B}$ immunoexpression. On the other hand, groups (2) and (5) revealed strong cytoplasmic and nuclear 
immunoexpression of NF- $\kappa \mathrm{B}$ in the surface palatal epithelium and malignant infiltrating epithelial cells and nests. Group (4) showed mild to moderate NF$\kappa \mathrm{B}$ cytoplasmic immunostaining.
Regarding the area\% and optical density, groups (2) and (5) showed the highest values among the studied groups with a highly significant difference (Table 1 and Figure 3).
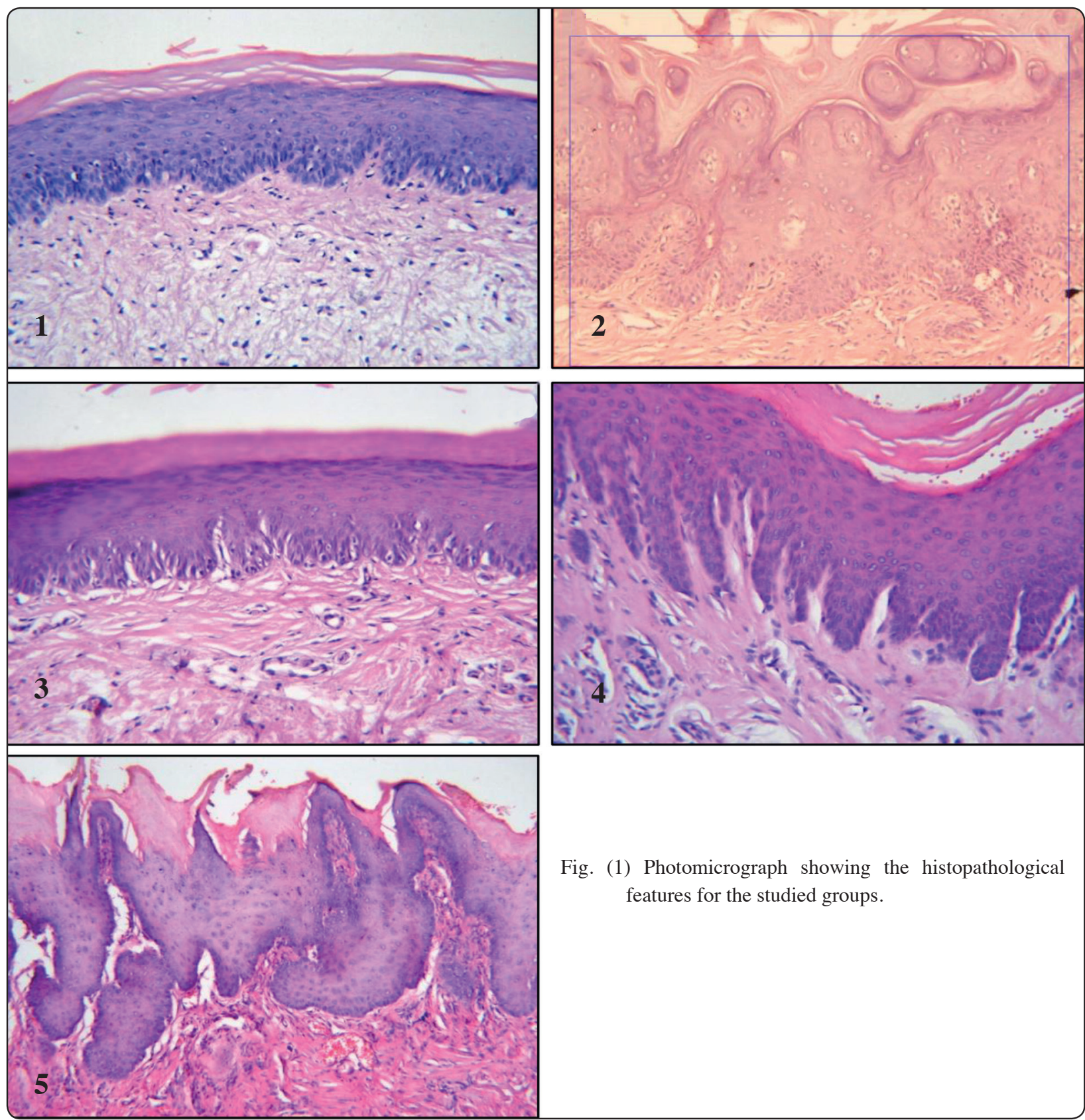

Fig. (1) Photomicrograph showing the histopathological features for the studied groups. 


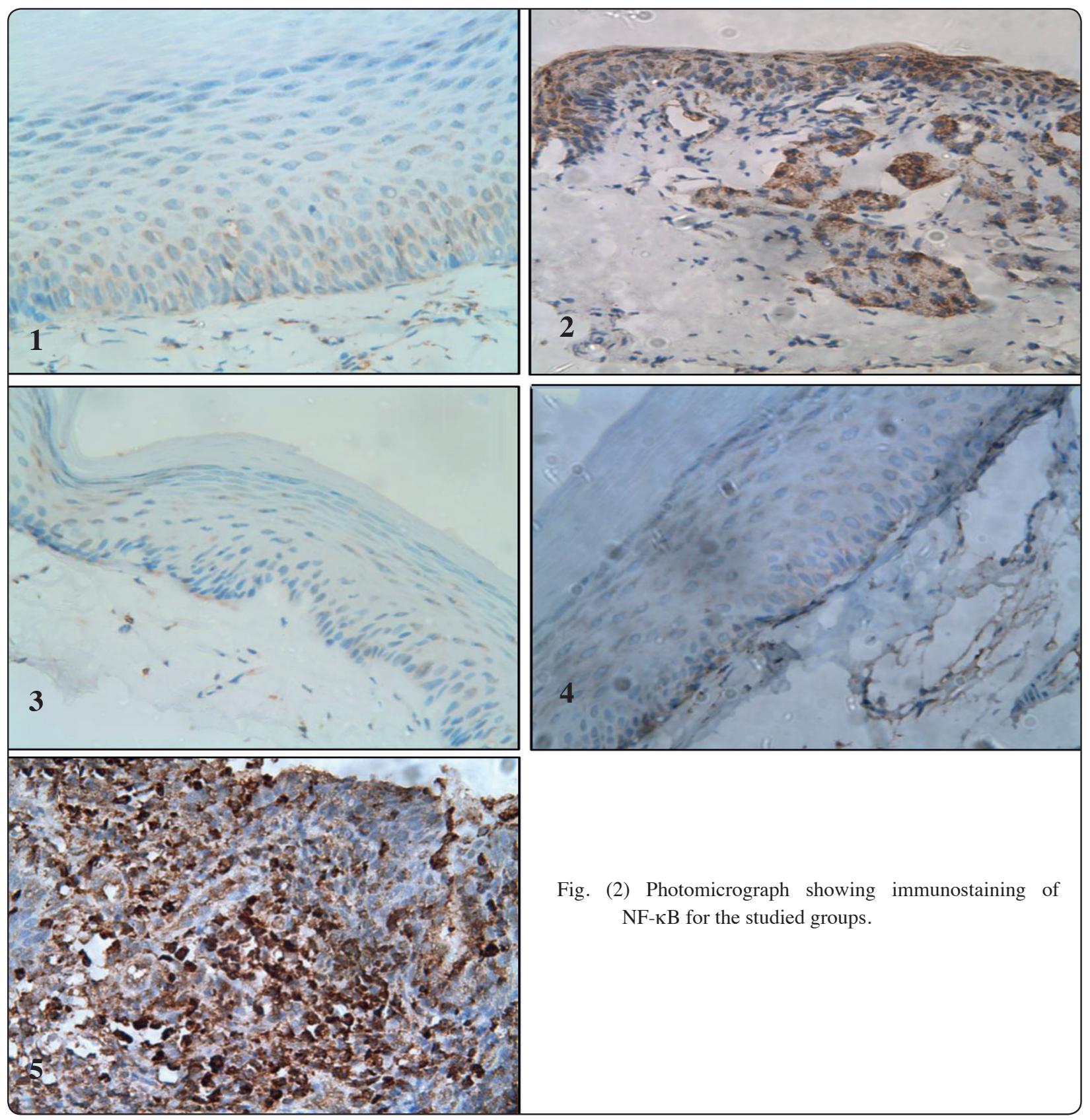

TABLE (1) Comparison NF-кb immunoexpression among the studied groups and its statistical significance using ANOVA test.

\begin{tabular}{|c|c|c|c|c|c|c|}
\hline \multirow{2}{*}{ Immuno-staining profile } & \multicolumn{5}{|c|}{ Mean \pm SD } & \multirow{2}{*}{ P-Value } \\
\cline { 2 - 6 } & Group 1 & Group 2 & Group 3 & Group 4 & Group 5 & \\
\hline Area \% & $3 \pm 0.101$ & $10.2 \pm 1.55$ & $2 \pm 0.2$ & $4 \pm 0.45$ & $9.8 \pm 1.44$ & $0.000^{*}$ \\
\hline Optical density & $22 \pm 0.37$ & $78.4 \pm 1.87$ & $26 \pm 0.34$ & $27 \pm 1.21$ & $76 \pm 1.6$ & $0.000^{*}$ \\
\hline
\end{tabular}

$*=$ significant $(P<0.05)$ 


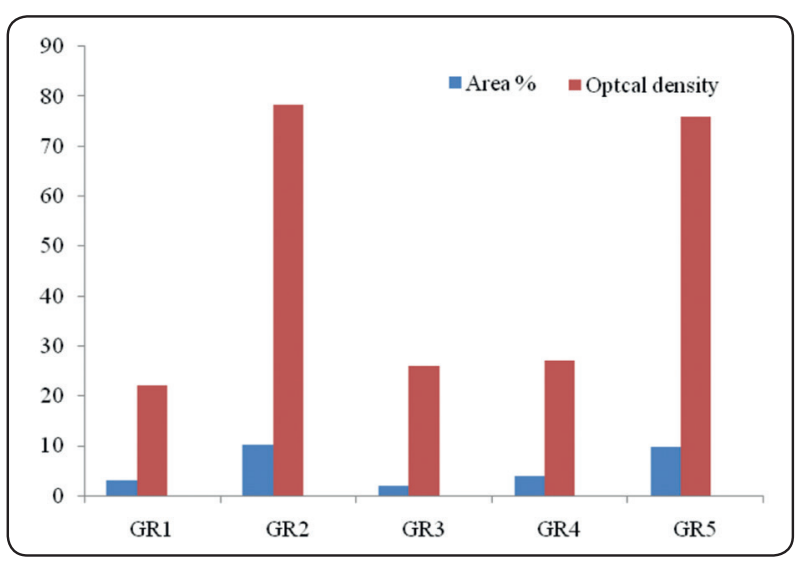

Fig (3) Bar chart illustrating the area $\%$ and optical density of the NF- $\kappa \beta$ immunoexpression in the studied groups.

\section{DISCUSSION}

Medicinal plants including garlic are receiving attention back again as chemopreventive and immunomodulating agents, thus providing new promising strategies in cancer therapy (Mirunalini et al., 2004; Larrosa et al, 2006; Arreola et al., 2015 and Baliga et al., 2015). DMBA induced experimental carcinogenesis is similar to human oral cancer at cellular and molecular level, thus it is ideal for evaluating the chemopreventive effect of medicinal plants (Manoharan et al., 2009 and Manoharan et al., 2016).

The histopathological results of the present study revealed that administration of garlic before and alternating with DMBA (group 4) is associated with the appearance of only some areas of hyperplasia with no signs of dysplasia except for basilar hyperplasia in 3 out of 15 animals (20\%). This was similar to Mirunalini et al. (2004) who reported that administration of garlic alternating with DMBA is associated with the appearance of hyperplastic lesions.

The current study showed that all rats that received DMBA alone (group 2) developed carcinoma, however on administration of garlic after DMBA (group 5) the number of rats that developed carcinoma decreased. This confirms the results of Balasenthil et al. (1999) who observed that administration of garlic after DMBA reduced the incidence of developing oral carcinoma.

As the histopathological results of groups (4) and (5) demonstrated that rats which received garlic before and alternating with DMBA, developed hyperplasia only, while some rats which received garlic after DMBA developed carcinoma, these results agree with Balasenthil \& Nagini (2000), The previous authors demonstrated that the administration of both DMBA and garlic together is associated with hyperplastic lesions only, however administration of garlic after DMBA is associated with hyperplasia, dysplasia and carcinoma. Accordingly, garlic exhibits more inhibitory effect on cancer development when it is applied before and alternating with cancer induction.

This was in accordance with Corpet and Tache (2002) who stated that garlic may have inhibitory effect on tumor formation in the initiation phase but not in the promotion phase. They confirmed that garlic decreased the formation of the preneoplastic lesions of colon during the initiation phase, but ineffective when administrated during promotion and progression phases.

Regarding NF- $\kappa \mathrm{B}$, the rats who received DMBA alone (group 2) and DMBA followed by garlic (group 5) showed the highest values of both area\% and optical density with strong cytoplasmic and nuclear immunoexpression.

These findings denote that the chemical carcinogen leads to activation of NF-אB pathway leading to squamous cell carcinoma formation and that garlic administration after DMBA in group (5) couldn't inhibit carcinogenesis efficiently. However, the inhibitory effect on NF-KB pathway was more obvious when garlic was administrated before and alternative with DMBA in group (4).

Our results were in accordance with Liu et al. (2006); Schäfer \& Kaschula (2014) and Arreola et al. (2015) who mentioned the role of garlic in maintaining the homeostasis of the immune system. They stated that some garlic derived compounds can 
inhibit NF- $\mathrm{BB}$ in white blood cells, macrophages, liver, skin and gingival fibroblasts.

Casey et al. (2015) confirmed that a significant end-point for assessment of the efficacy of cancer therapy is measuring changes in the tumor microenvironment including changes in targeted molecules. Thus, NF- $\kappa \mathrm{B}$ may be used in future as a sensitive end-point for evaluating the efficacy of therapeutics of oral cancer.

The observations of the present study confirmed that administration of aqueous garlic extract during the initiation period of DMBA-induced oral cancer in experimental animals could prevent cancer through inhibition of NF- $\mathrm{KB}$, where the chemopreventive and immunomodulatory effect of garlic are more pronounced during the early stages.

This may provide new insights into reducing the resistance of chemotherapy and radiotherapy through inhibition of NF- $\mathrm{KB}$ using naturally occurring micronutrient chemopreventive immunomodulatory agents to achieve synergistic effect during treatment.

\section{CONCLUSION}

- Garlic may be promising supplement in oral cancer therapy due to its chemopreventive immunomodulatory effects.

- NF-кB may serve as efficient molecular marker for assessment of the efficiency of medicinal plants in cancer therapy.

\section{Conflict of interest:}

No conflict of interest.

\section{REFERENCES}

1. Aggarwal S., Takada Y., Singh S., Myers J. and Aggarwal B.: Inhibition of growth and survival of human head and neck squamous cell carcinoma cells by curcumin via modulation of nuclear factor-kappa B signaling. Int J Cancer, 111(5): 679-692, 2004.

2. Arreola R., Quintero-Fabián S., López-Roa R., FloresGutiérrez E., Reyes-Grajeda J., Carrera-Quintanar L. and Ortuño-Sahagún D.: Immunomodulation and AntiInflammatory Effects of Garlic Compounds. Journal of Immunology Research, 2015: 1-13, 2015.

3. Balasenthil S. and Nagini S.: Inhibition of 7, 12-dimethylbenz [a] anthracene-induced hamster buccal pouch carcinogenesis by S-allylcysteine. Oral Oncol, 36(4): 382-386, 2000.

4. Balasenthil S., Arivazhagan S., Ramachandran CR., Nagini S.: Effects of garlic on 7, 12-Dimethylbenz[a] anthracene-induced hamster buccal pouch carcinogenesis. Cancer Detect Prev, 23(6): 534-538, 1999.

5. Balasenthil S., Rao K. and Nagini S.: Retinoic acid receptor- $\beta$ mRNA expression during chemoprevention of hamster cheek pouch carcinogenesis by garlic. Asia Pacific J ClinNutr, 12(2): 215-218, 2003.

6. Baliga KA., Kini R., Naik V. and Shravan Kini S.: Chemopreventive Agents; Its Role in Oral Cancer Prevention. Int J Adv Health Sci, 2 (1): 22-27, 2015.

7. Caseya SC., Amedeib A., Aquilano K. et al.: Cancer prevention and therapy through the modulation of the tumor microenvironment. Semin Cancer Biol, 35: s199-s223, 2015.

8. Corpet D. and Tache S.: Most effective colon cancer chemopreventive agents in rats: a systematic review of aberrant crypt foci and tumor data, ranked by potency. Nutr. Cancer, 43: 1-21, 2002.

9. El-Bayoumy K., Sinha R., Pinto J. and Rivlin R.: Cancer Chemoprevention by Garlic and Garlic-Containing Sulfur and Selenium Compounds. J Nutr, 136: 864-869, 2006.

10. Gupta S., Kim J., Prasad S. and Aggarwal B.: Regulation of survival, proliferation, invasion, angiogenesis, and metastasis of tumor cells through modulation of inflammatory pathways by nutraceuticals. Cancer Metastasis Rev, 29(3): 405-434, 2010.

11. Larrosa M., Tomás-Barberán F. and Espín J.: The dietary hydrolysable tannin punicalagin release ellagic acid that induces apoptosis in human colon adenocarcinoma Caco2 cells by using the mitochondrial pathway. J. Nutr. Biochem., 17: 611-625, 2006.

12. Lin C., Hsieh Y., Hsin C., Su C., Lin C., Wei L., Yang S. and Chien M.: Effects of NFkB1 and NFKBIA Gene Polymorphisms on Susceptibility to Environmental Factors and the Clinicopathologic Development of Oral Cancer. PLoS ONE, 7(4): e35078, 2012. 
13. Liu KL., Chen HW., Wang RY., Lei YP., Sheen LY., Lii CK.: DATS reduces LPS-induced iNOS expression, NO production, oxidative stress, and NF-kappa $b$ activation in RAW 264.7 macrophages. J Agric Food Chem, 54(9): 3472-3478, 2006.

14. Manoharan S., Singh R. and Balakrishnan S.: Chemopreventive Mechanisms of Natural Products in Oral, Mammary and Skin Carcinogenesis: An Overview. The Open Nutraceuticals Journal, 2: 52-63, 2009.

15. Manoharan S., Karthikeyan S., Essa MM., Manimaran A., Selvasundram R.: An overview of oral carcinogenesis. Int J Nutr Pharmacol Neurol Dis, 6: 51-62, 2016.

16. Mirunalini S., Kumaraguruparan R., Subapriya R. and Nagini S.: Garlic Oil Enhances Hepatic and Blood
Antioxidants During Hamster Buccal Pouch Carcinogenesis. Pharm Biol, 42 (3): 240-245, 2004.

17. Sarkar F., Li Y., Wang Z. andKong D.: NF-кB Signaling Pathway and Its Therapeutic Implications in Human Diseases. International Reviews of Immunology, 27: 293 319, 2008.

18. Schafer G. and Kaschula C.: The Immunomodulation and Anti-Inflammatory Effects of Garlic Organosulfur Compounds in Cancer Chemoprevention. Anti-Cancer Agents in Medicinal Chemistry, 14: 233-240, 2014.

19. Xiao G. and Fu J.: NF- $\kappa B$ and cancer: a paradigm of YinYang. Am J Cancer Res, 1(2): 192-221, 2011.

20. Williams H.: Molecular pathogenesis of oral squamous carcinoma. J Clin Pathol: Mol Pathol, 53: 165-172, 2000 